Supporting information

\title{
Bio-reduction of Chromate in a Methane-Based Membrane Biofilm Reactor
}

Chun-Yu Lai ${ }^{1}$, Liang Zhong ${ }^{1}$, Yin Zhang ${ }^{1}$, Jia-Xian Chen ${ }^{1}$, Li-Lian Wen ${ }^{1}$, Ling-Dong

$\mathrm{Shi}^{1}$, Yan-Ping Sun ${ }^{1}$, Fang $\mathrm{Ma}^{2}{ }^{2}$, Bruce E. Rittmann ${ }^{3}$, Chen $\mathrm{Zhou}^{3}$, Youneng Tang ${ }^{4}$, Ping Zheng ${ }^{1}$, He-Ping Zhao ${ }^{1,2, *}$

1. Department of Environmental Engineering, College of Environmental and

Resource Science, Zhejiang University, Hangzhou, China.

2. State Key Laboratory of Urban Water Resource and Environment, Harbin Institute of Technology, Harbin 150090, China.

3. Swette Center for Environmental Biotechnology, Biodesign Institute at Arizona State University, P.O. Box 875701, Tempe, Arizona 85287-5701, U.S.A.

4. Department of Civil and Environmental Engineering, FAMU-FSU College of Engineering, Florida State University, Tallahassee, Florida, 32310-6046, U.S.A.

* Correspondence to Dr. He-Ping Zhao. Tel: 0086-571-88982739, Fax:

0086-571-88982907, E-mail: zhaohp@,zju.edu.cn

Number of pages: 8

Number of tables: 1

Number of figures: 5 
The TEM analysis method:

The 5-cm-long section of the coupon fiber was then cut into smaller pieces of $\sim 0.5$

cm length for TEM (Hitachi H-7650, Japan) analysis following eight steps (SI).

1) Incubate the fiber samples with $2.5 \%$ glutaraldehyde in phosphate-buffered saline (PBS) for $12 \mathrm{~h}$ at $4^{\circ} \mathrm{C}$.

2) Encase the incubated samples with $1 \%$ agarose and repeat the incubation step.

3) Wash the encased fibers by PBS three times and fixe them in $1 \%$ osmium tetroxide in PBS for $1.5 \mathrm{~h}$.

4) After discarding the osmium tetroxide solution and washing the samples with PBS, dehydrate them by increased concentrations of ethanol $(30 \%, 50 \%, 70 \%$, $80 \%, 90 \%, 95 \%$ ethanol, and then twice at $100 \%$ ethanol), and then transfer them to absolute acetone for 20 min soaking.

5) Place samples into 1:1 or 3:1 mixture of embedding agent and acetone for $1 \mathrm{~h}$ or $2 \mathrm{~h}$, respectively, immerse tge samples in absolute embedding agent overnight, and then polymerize them at $70{ }^{\circ} \mathrm{C}$ for $12 \mathrm{~h}$.

6) Trim and thin-section the polymerized blocks using a LEICA EM UC7 ultra-microtome to get sections $70-90 \mathrm{~nm}$ thick, and stain these sections with uranyl acetate and alkaline lead citrate for 15 min respectively.

7) Load section samples on a TEM grid (Hitachi H-7650, Japan) and captur TEM images using a Gatan instrument (Gatan lns 832.H21WO, USA) at $80 \mathrm{kV}$.

8) Assay the elemental compositions using an EDS detector (EDAX GENESIS XM2, USA). 
Table S1. The average acceptor fluxes and methane-supply fluxes for each stage of the MBfR experiments

\begin{tabular}{|c|c|c|c|c|c|}
\hline \multirow[b]{2}{*}{ Stage } & \multicolumn{3}{|c|}{$\mathrm{Cr}(\mathrm{VI})$} & \multicolumn{2}{|c|}{ Electron donor $\left(\mathrm{CH}_{4}\right)$} \\
\hline & Surface loading & Flux & Electron donor consumed & $\begin{array}{l}\text { Maximum } \mathrm{CH}_{4} \\
\text { flux }\end{array}$ & Actual $\mathrm{CH}_{4}$ flux \\
\hline & $\left(\mathrm{mg} / \mathrm{m}^{2}-\mathrm{d}\right)^{\mathrm{a}}$ & $\left(\mathrm{mg} / \mathrm{m}^{2}-\mathrm{d}\right)^{\mathrm{b}}$ & $\left(\mathrm{mmol} \mathrm{CH}_{4} / \mathrm{m}^{2}-\mathrm{d}\right)$ & $\left(\mathrm{mmol} \mathrm{CH}_{4} / \mathrm{m}^{2}-\mathrm{d}\right)^{\mathrm{c}}$ & $\left(\mathrm{mmol} \mathrm{CH} / \mathrm{m}^{2}-\mathrm{d}\right)$ \\
\hline 1 & 128.8 & 68.0 & 0.98 & 57.9 & 0.99 \\
\hline 2 & 365.9 & 37.6 & 0.54 & 57.9 & 0.55 \\
\hline 3 & 119.3 & 109.2 & 1.57 & 57.9 & 1.58 \\
\hline 4 & 233.5 & 231.3 & 3.33 & 57.9 & 3.34 \\
\hline 5 & 377.9 & 367.9 & 5.30 & 57.9 & 5.31 \\
\hline
\end{tabular}

a: Calculated from equation 1. b: Calculated from equations 2 through 3. c: Maximum $\mathrm{CH}_{4}$ flux was calculated according to Tang et al (2012), considering the $\mathrm{Pm}$-if $\left(\mathrm{CH}_{4}\right.$ pressure at the interface of membrane and liquid film) was $0 . \quad 1 \mathrm{bar}=14.5 \mathrm{psig}$ 


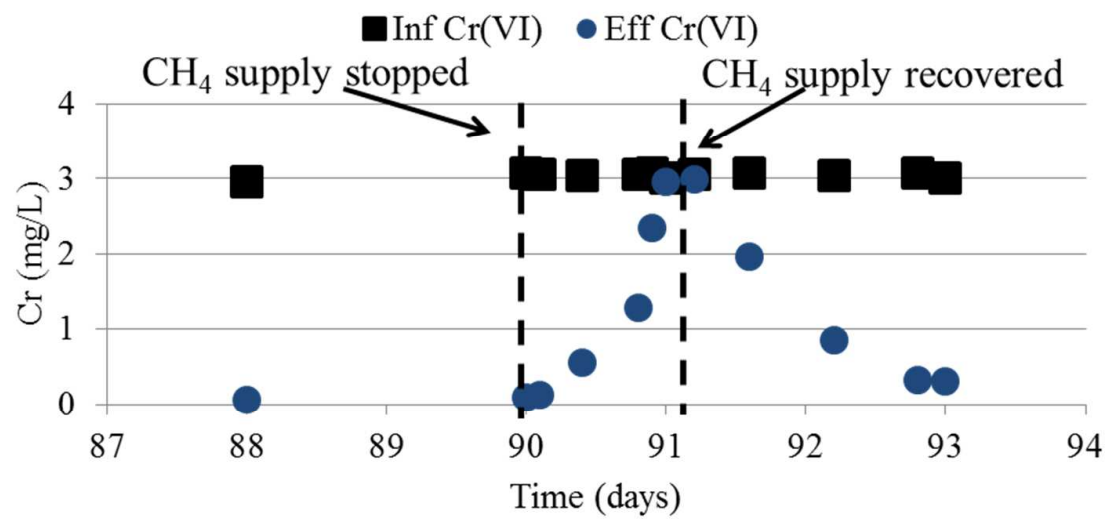

Figure S1. Measures of $\mathrm{Cr}(\mathrm{VI})$ reduction in the $\mathrm{CH} 4$-based $\mathrm{MBfR}$ when $\mathrm{CH}_{4}$ supply was stopped and recovered. 


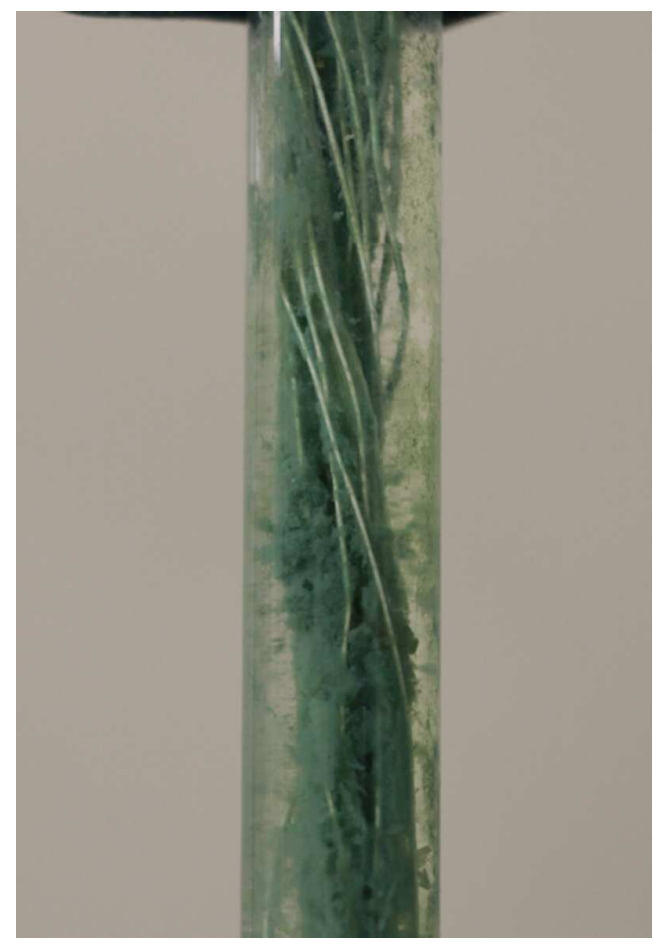

Figure S2. Photograph of the main fiber bundles in the MBfR taken at the end of MBfR operation. The sage green solids are attributable to immobilized $\mathrm{Cr}(\mathrm{III})$, particularly $\mathrm{Cr}(\mathrm{OH})_{3} \cdot{ }^{50}$ 


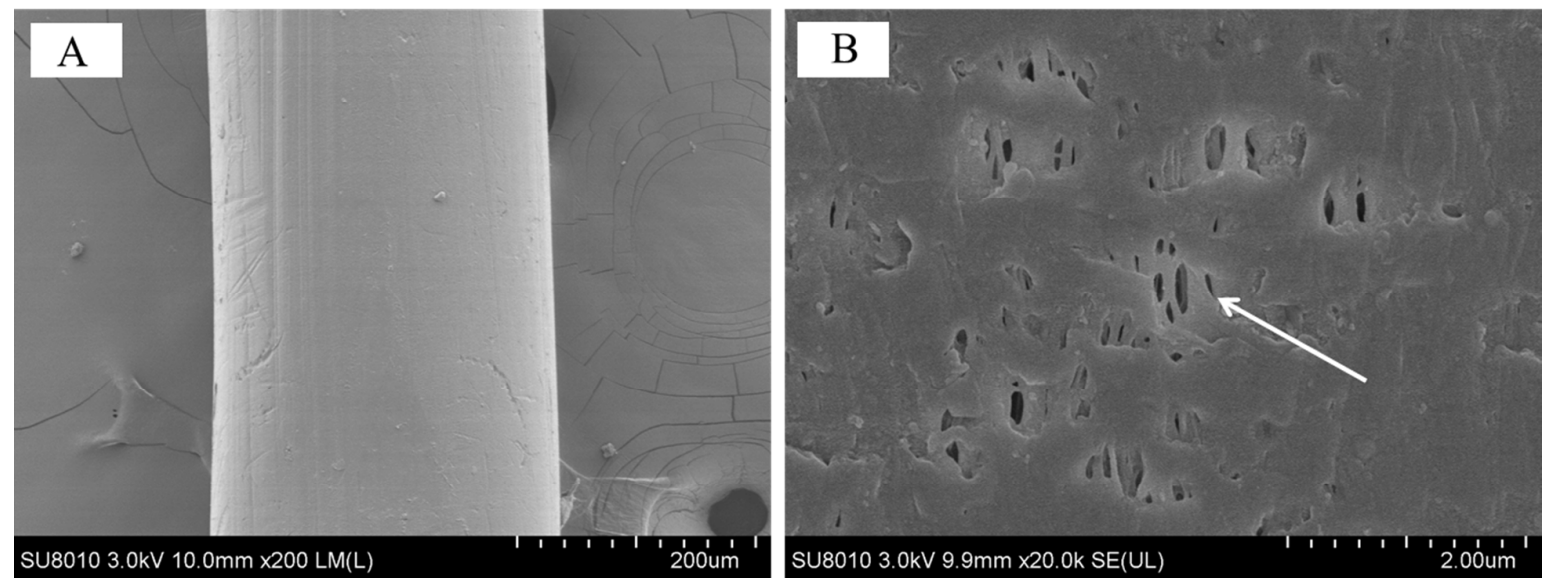

Figure S3. SEMs of hollow fiber membrane before inoculation. SEM was

observed at 200 (A) and 20000 (B) times magnification. White arrow indicates the micropores of the fiber. 

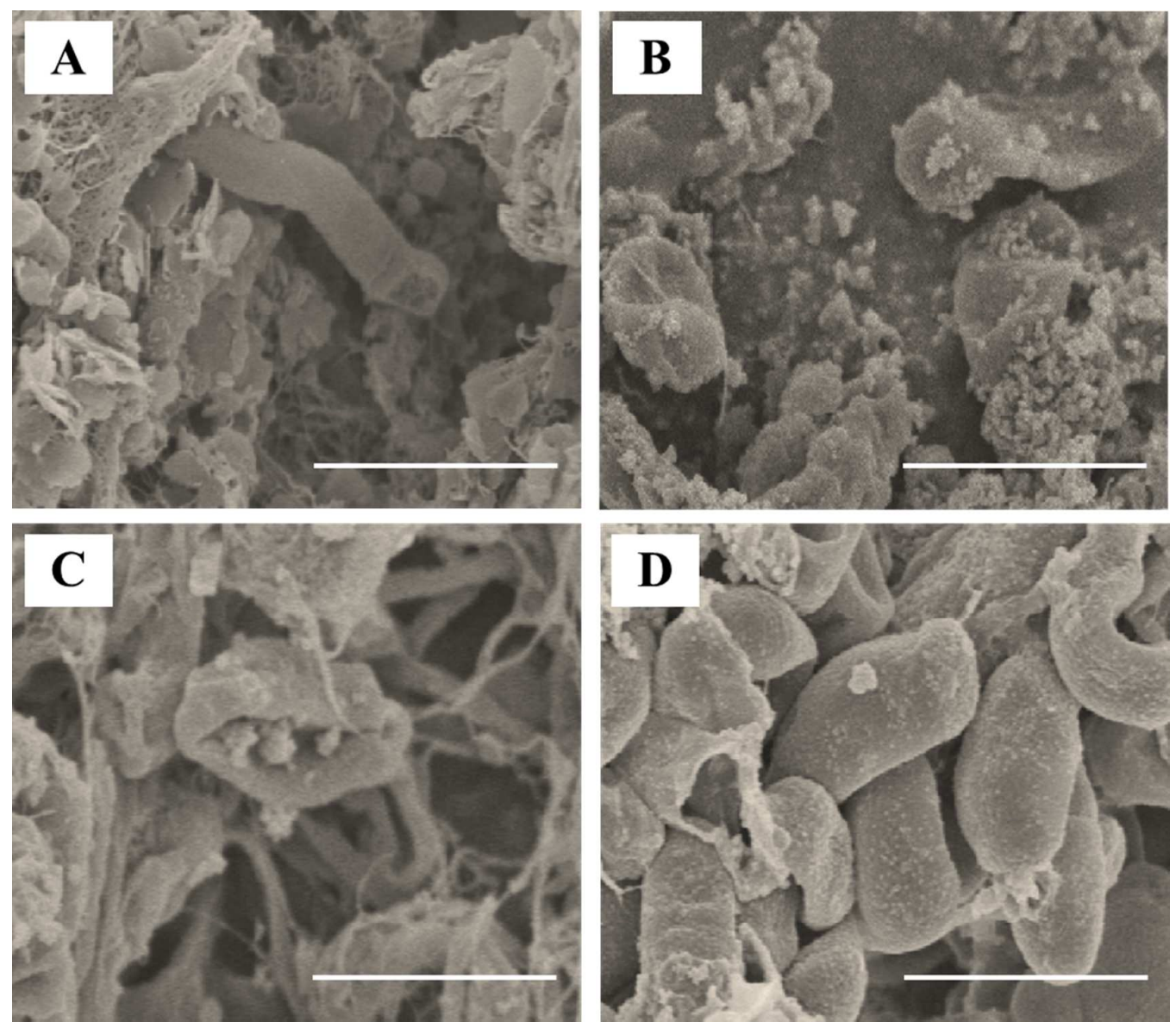

Figure S4. Magnified SEM imagines of inoculum (A), Stages 1 (B), 2 (C), and 5 (D) corresponding to Figure 2. 


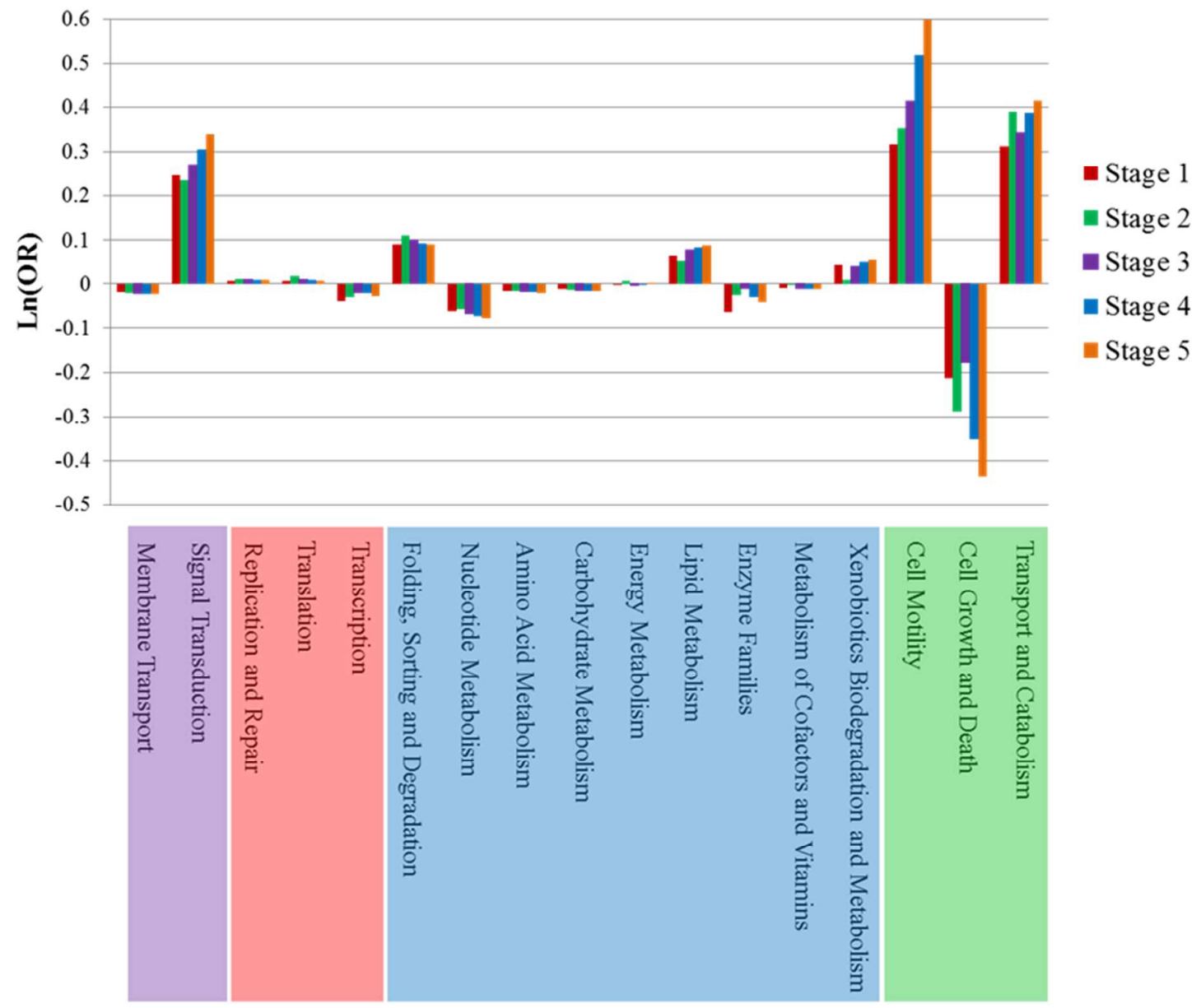

Figure S5. Odds ratios of predictive functions assigned by KEGG category database in biofilms (stages 1-5) compared to those in the inoculated culture. The functions of human diseases and organismal systems (all $<1 \%$ in abundance) are not included. 